\title{
PERFIL DE PESSOAS ACOMETIDAS POR DIABETES MELLITUS
}

\author{
PROFILE OF PEOPLE AFFECTED BY DIABETES MELLITUS
}

\author{
Cristiana Barbosa da Silva Gomes ${ }^{1}$ \\ Rosângela Vidal de Negreiros ${ }^{2}$ \\ Rachel Hellen Monteiro da Costa ${ }^{3}$ \\ Larissa Queiroz de Oliveira ${ }^{4}$
}

\begin{abstract}
RESUMO: OBJETIVO: descrever o perfil de pessoas acometidas por Diabetes Mellitus (DM) tipo 2, cadastrados no programa HIPERDIA. METODOLOGIA: Tratase de um estudo descritivo, exploratório, com abordagem quantitativa, que caracteriza-se por um levantamento de dados disponíveis no Sistema DATASUS do Ministério da Saúde, onde foi realizada uma análise criteriosa, estabelecendo informações epidemiológicas e de morbidade do programa HIPERDIA, no Estado da Paraíba, entre 2009 e 2013, abordando especificamente o Diabetes Mellitus tipo 2. RESULTADOS: No período analisado (2009-2013), houve 3.358 casos de Diabetes mellitus Tipo 2 na Paraíba. O ano de 2011 teve 988 casos entre todas as idades, em relação a faixa etária a maior ocorrência foi entre 40 e 59 anos de idade com 1.601 casos $(47,6 \%)$, o gênero feminino teve destaque com 2.020 casos $(60,1 \%)$; Como fatores de risco, foi observado que a maioria não era tabagista 2658 (79,1\%). Foi destaque ainda o fato de 2166 casos $(64,5 \%)$ não apresentarem sobrepeso, quanto às complicações associadas ao DM, apresentou maior frequência de pé diabético com 91 casos, equivalente a $(2,75 \%)$ dos acometidos por DM tipo 2. No entanto, amputação de pé diabético ocupou a menor frequência com 65 casos $(1,95 \%)$. CONCLUSÃO: A divulgação das informações referentes à saúde para a população, estabelecidas nas plataformas científicas, caracteriza-se como o primeiro passo, para que a saúde pública faça de doenças como o DM tipo 2, passíveis de controle, dentre as Doenças Crônicas Não Transmissíveis de maior evidência na população.
\end{abstract}

\footnotetext{
1 Enfermeira Graduada pela Universidade Federal de Campina Grande - UFCG / e-mail: redentor.cristiana@gmail.com.

2 Professora orientadora: Doutoranda da USP. Mestre em Enfermagem. Docente do curso de Enfermagem do CCBS/UFCG. E-mail: negreiros.vidal@hotmail.com.

${ }^{3}$ Graduanda do Curso de Enfermagem da Universidade Federal de Campina Grande - UFCG, E-mail: rachel09hellen@gmail.com.

${ }^{4}$ Graduanda do Curso de Enfermagem da Universidade Federal de Campina Grande - UFCG, E-mail: larissaq12.1q@gmail.com.
} 
Palavras chave: Autocuidado. Diabetes mellitus tipo 2. Pé diabético. Qualidade de vida.

RESUME: OBJECTIVE: to describe the profile of people affected by type 2 Diabetes Mellitus (DM), registered in the HIPERDIA program. METHODOLOGY: This is a descriptive, exploratory study with a quantitative approach, which is characterized by a survey of data available in the DATASUS System of the Ministry of Health, where a careful analysis was carried out, establishing epidemiological and morbidity information from the HIPERDIA program., in the State of Paraíba, between 2009 and 2013, specifically addressing Type 2 Diabetes Mellitus. RESULTS: In the period analyzed (2009-2013), there were 3,358 cases of Type 2 Diabetes mellitus in Paraiba. The year 2011 had 988 cases among all ages, in relation to the age group the highest occurrence was between 40 and 59 years of age with 1,601 cases (47.6\%), the female gender stood out with 2,020 cases (60.1\%); As risk factors, it was observed that the majority was not a smoker 2658 (79.1\%). It was also highlighted the fact that 2166 cases (64.5\%) were not overweight, as for the complications associated with DM, presented a higher frequency of diabetic foot with 91 cases, equivalent to (2.75\%) of those affected by type 2 DM. However, diabetic foot amputation was the least frequent with 65 cases (1.95\%). CONCLUSION: The dissemination of health-related information to the population, established on scientific platforms, is characterized as the first step, so that public health can make diseases like type 2 DM, subject to control, among Chronic Non- Communicable Diseases most evident in the population.

Keywords: Self-care. Type 2 diabetes mellitus. Diabetic foot. Quality of life. 


\section{INTRODUÇÃO}

Entender o processo de adoecimento da humanidade vai muito além da observação de sinais e sintomas determinados pela fisiopatologia. Adoecer significa apresentar vulnerabilidade dentro das dimensões da individualidade, ou seja, é possível que um indivíduo tenha parâmetros biológicos, prognóstico e implicações para tratamento semelhante a tantos outros e mesmo assim ser afetado de forma distinta, resultando em diferentes manifestações de sintomas e desconforto, sendo comprometido de forma diferenciada (VIANNA, 2012).

Nesse sentido, é necessário não desconsiderar as divergências, mas buscar nas semelhanças os padrões de adoecimento e, consequentemente, poder vislumbrar possibilidades de tratamento que alcance as diversas populações e até mesmo poder antecipar- se aos agravos de modo preventivo na atenção a saúde.

Levando em consideração os padrões de adoecimento social é importante destacar as Doenças Crônicas Não Transmissíveis (DCNT), definida como o conjunto de condições crônicas relacionadas a causas múltiplas de início gradual, prognóstico usualmente incerto, com longa ou indefinida duração, apresenta mudança com o tempo, podendo gerar incapacidades. Esta por sua vez vem ganhando grande espaço onde antes era ocupado pelas doenças infecciosas, principalmente nos países em desenvolvimento onde alcançará uma estimativa de 80\% da carga de doenças no ano de 2020 (BRASIL, 2013).

Caracterizada como uma DCNT o Diabetes Mellitus (DM) é uma doença metabólica, multifatorial, que ocorre devido a falta de insulina e/ou diminuição dela no organismo. Para classificação, existe o Tipo 1, Tipo 2, DM gestacional e alguns casos específicos. O DM2 é responsável pelo acometimento de 80 a $90 \%$ de todos os casos. Como fatores de risco é possível destacar idade acima de 45 anos, excesso de peso, sedentarismo, histórico familiar de DM, hipertensão arterial, alterações das taxas de colesterol e triglicerídeos. 
O DM alcançou proporções epidêmicas com estimativa de 450 milhões de pessoas que sofrem dessa doença ao longo do mundo e esse fato tem causado impacto importante sobre a saúde dos afetados, tais como complicações vasculares, aumento da morbidade, redução da qualidade de vida, e elevação da taxa de mortalidade. As causas podem ser de ordem genética, biológica e ambiental e não são completamente conhecidos. Em 2017, a Federação Internacional de Diabetes estimou que $8,8 \%$ da população mundial com 20 a 79 anos de idade (424,9 milhões de pessoas) viviam com diabetes se as tendências persistirem o quantitativo de pessoas com diabetes em 2045 será de 628,6 milhões (SBD, 2019).

De acordo com Tonetto et. al (2018), o Brasil, em 2017, ocupava o quarto lugar entre os 10 países com maior número de acometidos por DM, aproximadamente, 12,5 milhões de pessoas e apresentava-se entre as cinco principais causas de morte, responsável por mais de 59 mil óbitos em 2015.

Segundo o Ministério da Saúde, entre os anos de 2010 e 2016, 12.444 pessoas foram a óbito na Paraíba em consequência do diabetes, houve um crescimento desses dados em 8,4\% no período, passou então de 1.698 para 1.841 casos de mortes em 2017. Na contrapartida dos óbitos, as internações caíram 16,1\%: foram 3.620 em 2010 e 3.037, em 2016 (BRASIL, 2018).

Diante da situação geral de DM é importante ressaltar que a promoção de hábitos saudáveis atrelados à prevenção é sem dúvidas a melhor opção para o controle desse agravo. Para isso faz-se necessário conhecer a população acometida para que possa ser acompanhada, como o percentual de saudáveis, para que seja possível levar conhecimento a todos e prevenir o aumento de casos.

O interesse em desenvolver esta pesquisa partiu da inquietude em avaliar 0 perfil dos acometidos por DM tipo 2 no estado da Paraíba entre 2009 e 2013, cadastrados no programa HIPERDIA.

A relevância do estudo se estabelece pelo conhecimento dessa população, podendo a partir daí divulgar tais dados dentro da comunidade acadêmica, na perspectiva de tal conhecimento garantir aprendizado, conduzir as equipes de saúde a estabelecer novas metas dentro das dificuldades encontradas, de forma a prevenir novos casos e melhorar a qualidade de vida daqueles que já sofrem com a doença, bem como, desenvolver ações que atenda a demanda expressa pelos profissionais 
de saúde, reconhecendo com isso, novas ferramentas mediadoras para mover ações de cuidados, visando planejar, monitorar e avaliar estratégias de combate ao avanço do DM tipo 2.

Levando em consideração o DM tipo 2 como um grande e importante problema de saúde pública e fazendo uso da promoção da saúde em prol da prevenção de novos casos e acompanhamento dos casos já existentes diante das possíveis complicações de potencialidades fatais. O objetivo desse estudo é descrever o perfil das pessoas acometidas por Diabetes mellitus tipo 2, cadastrados no programa HIPERDIA no período de 2009 a 2013, na Paraíba, de acordo com o DATASUS do Ministério da Saúde, Brasil.

\section{METODOLOGIA}

Trata-se de um estudo descritivo, exploratório, com abordagem quantitativa. Esse estudo resulta de um levantamento de dados disponíveis no Sistema DATASUS do Ministério da saúde - Brasil, onde foi feito uma análise criteriosa, estabelecendo informações epidemiológicas e de morbidade do programa HIPERDIA, no Estado da Paraíba, entre 2009 e 2013, abordando especificamente o Diabetes Mellitus tipo 2.

Levando em consideração o Estado da Paraíba, apresenta um território de $56.467,239 \mathrm{~km}^{2}$, com uma população estimada para o ano de 2019 de 4.018,127 pessoas distribuídas em 223 municípios.

Foi levando em consideração na busca de dados da Biblioteca Virtual em Saúde (BVS), utilização dos descritores: diabetes mellitus tipo 2, autocuidado, complicações do diabetes, qualidade de vida, pé diabético e morbidade e como critério de inclusão, artigos completos em português, com publicação nos últimos 5 anos. Após leitura dinâmica, foi obtido como critério de exclusão os artigos que se repetiam e aqueles que não condiziam com a temática, obtendo ao fim 28 artigos completos, desses, foi realizado uma segunda leitura criteriosa direcionando a 
pesquisa ao tema proposto. Os dados da pesquisa foram exportados em fevereiro de 2020, referentes ao período da pesquisa.

\section{RESULTADOS}

No período analisado (2009-2013), houve 3.358 caso de DM Tipo 2 na Paraíba. O ano de maior ocorrência registrada foi em 2011 com 988 casos entre todas as idades, em relação a faixa etária com maior ocorrência foi entre 40 e 59 anos de idade com 1.601 casos $(47,6 \%)$, seguido dos maiores de 60 anos com 1.255 casos $(37,3 \%)$, fato exibido na (tabela 1$)$.

Tabela1 - Distribuição de Diabetes mellitus Tipo 2, por faixa etária e ano de ocorrência na Paraíba, de 2009-2013.

\begin{tabular}{|c|c|c|c|c|c|c|c|c|c|}
\hline \multirow[t]{2}{*}{ Ano } & \multicolumn{2}{|c|}{ Até 19 anos } & \multicolumn{2}{|c|}{20 a 39 anos } & \multicolumn{2}{|c|}{40 a 59 anos } & \multicolumn{2}{|c|}{$\geq 60$ anos } & \multirow{2}{*}{$\frac{\text { Total }}{\mathbf{N}}$} \\
\hline & $n$ & $\%$ & $\mathbf{n}$ & $\%$ & $n$ & $\%$ & $n$ & $\%$ & \\
\hline 2009 & 15 & 1,6 & 112 & 12,4 & 409 & 45,5 & 361 & 40,2 & 897 \\
\hline 2010 & 8 & 1 & 108 & 13,9 & 364 & 47,1 & 292 & 37,8 & 772 \\
\hline 2011 & 12 & 1,2 & 134 & 13,5 & 485 & 49 & 357 & 36,1 & 988 \\
\hline 2012 & 5 & 0,8 & 99 & 15,9 & 306 & 49,1 & 212 & 34 & 622 \\
\hline 2013 & 3 & 3,7 & 6 & 7,5 & 37 & 46,8 & 33 & 41,7 & 79 \\
\hline Total & 43 & 1,2 & 459 & 13,6 & 1601 & 47,6 & 1255 & 37,3 & 3358 \\
\hline
\end{tabular}

Fonte: Ministério da saúde, Sistema de cadastramento de hipertensos e diabéticos HIPERDIA. Dados exportados em Fevereiro de 2020.

Em relação ao gênero, o feminino teve destaque com 2.020 casos $(60,1 \%)$, evidenciado na (tabela 2). 
Tabela 2 - Ocorrência de Diabetes Mellitus Tipo 2 por gênero na Paraíba, de 20092013.

\begin{tabular}{lcc}
\hline Diabetes Mellitus Tipo 2 & & \\
\multicolumn{1}{c}{ Gênero } & $\mathbf{n}$ & $\%$ \\
\hline Feminino & 2020 & 60,1 \\
Masculino & 1338 & 39,8 \\
Total & 3358 & 100 \\
\hline
\end{tabular}

Fonte: Ministério da saúde, Sistema de cadastramento de hipertensos e diabéticos HIPERDIA. Dados exportados em Fevereiro de 2020.

Como fatores de risco, foi observado que a maioria não era tabagista 2658 $(79,1 \%)$. Foi destaque ainda o fato de 2166 casos (64,5\%) não apresentarem sobrepeso (tabela 3).

Tabela 3 - Diabetes mellitus Tipo 2 por fatores de risco e Complicações na Paraíba, de 2009- 2013.

\begin{tabular}{lcccc}
\hline \multirow{2}{*}{ Variáveis } & \multicolumn{4}{c}{ Diabetes Mllitus Tipo 2 } \\
\cline { 2 - 5 } Tabagista & Sim & $\%$ & Não & $\%$ \\
Sedentário & 700 & 20,8 & 2658 & 79,1 \\
\hline Sobrepeso & 1520 & 45,2 & 1838 & 54,7 \\
& 1192 & 35,4 & 2166 & 64,5 \\
Complicações & Sim & $\%$ & Não & $\%$ \\
Doença renal & 68 & 2 & 3290 & 97,9 \\
Acidente vascular cerebral & 76 & 2,2 & 3282 & 97,7 \\
Infarto agudo do miocárdico & 74 & 2,2 & 3284 & 97,7 \\
Pé diabético & 91 & 2,7 & 3267 & 97,2 \\
Amputação de pé diabético & 65 & 1,9 & 3293 & 98 \\
\hline
\end{tabular}

Fonte: Ministério da saúde, Sistema de cadastramento de hipertensos e diabéticos HIPERDIA. Dados exportados em Fevereiro de 2020. 
Em relação às complicações associadas ao $\mathrm{DM}$, apresentou maior frequência de pé diabético com 91 casos, equivalente a (2,75\%) dos acometidos por DM tipo 2. No entanto, a amputação de pé diabético ocupou a menor frequência com 65 casos $(1,95 \%)$, (tabela 3$)$.

\section{DISCUSSÃO}

O Diabetes é um problema de saúde pública com proporções epidêmicas. O Brasil, que ocupava a $8^{a}$ posição, com prevalência de 4,6\%, em 2000, passará para a $6^{a}$ posição em 2030 com 11,3\%, de acordo com a estimativa da Organização Mundial de Saúde em relação ao número de casos de diabetes (BRASIL, 2013).

No Brasil, ocorreram 51.828 mortes por diabetes, em 2009, o DM e a Hipertensão Arterial Sistêmica (HAS), são responsáveis pela primeira causa de morte e hospitalizações no Sistema Único de Saúde (SUS). As complicações da doença sejam elas agudas e/ou crônicas causam alta morbidade e consequentemente leva a altos custos para os sistemas de saúde. Gastos relacionados ao diabetes em todo o mundo, em 2010, foram estimados em 11,6 \% do total dos gastos com a saúde, o Brasil apresenta dados semelhantes (BRASIL, 2013).

Essa caracterização de custos pode ser apontada como estratégia fundamental na elaboração de novas ações que possam mudar o processo de saúde/doença frente aos mais diversos serviços de saúde, seja ele primário, secundário ou terciário dentro das Redes de Atenção a Saúde que possam mobilizar a prestação de cuidados específicos a pacientes diabéticos.

Com relação aos dados apresentados, o ano com maior quantitativo de pessoas acometidos pela doença foi 2011 com 988 casos. É possível observar que a faixa etária com maior número de casos foi a de 40 a 59 anos de idade com um total de 1.601 casos (47,6\%) fato que diverge do estudo de Flor e Campos (2017), onde relatam que a maioria no seu estudo são acometido por DM adultos acima de 60 anos de idade. Porém, de acordo com os dados apresentados na tabela 1, o 
maior número de casos está na população acima de 40 anos o que se enquadra perfeitamente com o a linha de rastreamento do Ministério da saúde, onde o indicativo de rastreio frente a idade apresentar-se acima de 45 anos junto a outros fatores (BRASIL, 2006).

Em se tratando do gênero, o feminino é o grande destaque com 2.020 casos $(60,1 \%)$ da amostra, Brasil (2013) refere que entre os anos de 2006 e 2011 houve um aumento de casos de DM entre os homens que passou de 4,4\%, em 2006, para 5,2 \%, em 2011, mesmo assim as mulheres apresentaram uma proporção maior, correspondendo a $6 \%$ dessa população.

Em relação as varáveis investigadas, o sedentarismo com 1520 casos $(45,2 \%)$ e o sobrepeso 1192 casos $(35,4 \%)$ tiveram destaque na amostra, uma vez que, além de ser considerado fator de risco para desenvolver a doença, são fatores de clara importância para o controle glicêmico e consequentemente, o controle do DM. Esse fato corrobora com o estudo de Lima et.al (2017), onde é possível observar que as variáveis investigadas nesse estudo são também tradadas naquele estudo como fatores de risco de grande destaque para o desenvolvimento do DM, o estudo ainda citou que esse fato estende-se para o Brasil inteiro e destacou-se até mesmo internacionalmente.

Sobre o tabagismo, 2658 pessoas $(79,1 \%)$ acometidas por DM, relataram não fumar, no entanto, ainda foram encontradas no período do estudo 700 fumantes $(20,8 \%)$, o que é bastante preocupante. O tabaco possui como constituinte a nicotina que produz alto impacto no organismo, principalmente no sistema cardiovascular que por sua vez, tem os vasos periféricos afetados, bem como, aumento da pressão arterial e da frequência cardíaca A nicotina também provoca o aumento da Lowdensity lipoprotein (LDL) e diminui a quantidade da High-density lipoprotein (HDL) disponíveis no organismo. Esse comprometimento cardiovascular a fisiopatologia do DM2, pode causar complicações severas no paciente (HOCAYEN, MANECK MALFATTI; 2010).

Em relação às complicações apresentadas pela amostra o pé diabético foi destaque 91 casos (2,7\%), desses, 65 (1,9\%) tiveram amputação em decorrência ao pé diabético. Segundo Dutra et al. (2017), a incidência de indivíduos diabéticos 
desenvolverem complicações relacionadas ao pé diabético é de 15 a $25 \%$, e a cada minuto, três amputações ocorrem no mundo em consequência do DM.

A dor em decorrência da neuropatia periférica costuma ser de moderada a grave e geralmente se manifesta com maior intensidade a noite causando distúrbios do sono, também pode ser motivo para interrupções de atividades sociais de laser e, consequentemente está bastante atrelada a depressão (DUTRA et al; 2017).

\section{CONCLUSÃO}

De acordo com os dados apresentados no presente estudo, o DM repete números alarmantes também no estado da Paraíba, mostrou-se um problema de saúde pública com consequências alarmantes e preocupantes para toda a comunidade de saúde. É possível observar que os gastos com a recuperação dos acometidos é bastante considerável principalmente para os países em desenvolvimento.

O ano de 2011 foi o que teve mais casos de DM2 na Paraíba, seguido por uma queda em 2012, no entanto, o número de casos mostrou-se em destaque entre o público feminino. A obesidade e sedentarismo apresentaram-se de forma bastante considerável em seus números, revelando uma população doente e com descontrole da doença já estabelecida; a principal complicação (Pé diabético) pode ser considerada um fator limitante dentro da fisiopatologia da neuropatia periférica, trazendo ao paciente consequências biopsicossocias de grande importância, que têm impactado de forma a levar muitos pacientes à depressão.

É importante ressaltar, diante dos dados apresentados, o DM2 é uma doença de difícil controle, no entanto, a prevenção é considerada estrategia importante diante dos dados apresentados nesse estudo.

Nesse sentido, a divulgação das informações de saúde para a população, estabelecidas nas plataformas científicas, é o primeiro e mais importante passo, para que a saúde pública faça de doenças como o Diabetes mellitus tipo 2 passível de controle e regressão de números de casos, fazendo com que haja uso das políticas 
públicas dentro das Redes de atenção saúde e, considerando a portas de entrada do SUS, em especial, a Atenção primária.

\section{REFERÊNCIAS BIBLIOGRÁFICAS}

ARAUJO FILHO, Augusto Cezar Antunes de et al. Perfil epidemiológico do diabetes mellitus em um estado do nordeste brasileiro Epidemiological profile of Diabetes Mellitus in a northeastern brazilian state. Revista de Pesquisa: Cuidado é Fundamental Online, [S.I.], v. 9, n. 3, p. 641647, july 2017.

BRASIL. Ministério da Saúde. Portal do Ministério da Saúde. Sistema de informação de saúde DATASUS. Programa de cadastramento e acompanhamento de hipertensos e diabéticos. 2020.

Ministério da Saúde. Secretaria de Atenção à Saúde. Departamento de Atenção Básica. Diabetes Mellitus / Ministério da Saúde, Secretaria de Atenção à Saúde, Departamento de Atenção Básica. - Brasília: Ministério da Saúde, 2013.

. Ministério da Saúde. Secretaria de Atenção a Saúde. Departamento de Atenção Básica. Estratégia para o cuidado da pessoa com doença crônica: diabetes mellitus. Brasília (DF): Ministério da Saúde; 2013.

Ministério da Saúde. Universidade Federal de São Paulo. Pró Reitoria de Extensão.

Processo saúde-doença. Universidade Aberta do SUS- UNA SUS. Governo Federal, 2012.

Ministério da Saúde. Secretaria de Atenção à Saúde. Departamento de Atenção Básica. Diabetes Mellitus / Ministério da Saúde, Secretaria de Atenção à Saúde, Departamento de Atenção Básica. - Brasília: Ministério da Saúde, 2006.

DUTRA, Luz Marina Alfonso et al. Avaliação do risco de ulceração em indivíduos diabéticos. Rev. Bras. Enferm. Brasília , v. 71, supl. 2, p. 733-739, 2018.

FLOR, Luisa Sorio; CAMPOS, Monica Rodrigues. Prevalência de diabetes mellitus e fatores associados na população adulta brasileira: evidências de um inquérito de base populacional. Rev. bras. epidemiol. São Paulo , v. 20, n. 1, p. 16-29, Mar. 2017.

\section{FUNDAÇÃO INSTITUTO BRASILEIRO DE GEOGRAFIA E ESTATÍSTICA. Censo demográfico. $\quad 2008 . \quad$ Disponível em: <http://www.ibge.gov.br/estadosat/temas.php?sigla=pb\&tema=censodemog2010_amostra>.}

HOCAYEN, Palloma Almeida Soares; MANECK MALFATTI, Carlos Ricardo. Tabagismo em pacientes diabéticos: predisposição às doenças crônico-degenerativas e neoplasia. Cinergis, Santa Cruz do Sul, v. 11, n. 2, set. 2011.

LIMA, Carla Lidiane Jácome de et al . Characterization of users at risk of developing diabetes: a cross-sectional study. Rev. Bras. Enferm. Brasília, v. 71, supl. 1, p. 475-482, 2018.

Organização Mundial de Saúde. OMS. Atividade física [Internet]. 2016 [cited 2016 Aug 25]. Available from: http://www.who.int/mediacentre/factsheets/fs385/es/.

Sociedade Brasileira de Diabetes. Diretrizes da Sociedade Brasileira de Diabetes: 2019-2020. 
São Paulo: AC Farmacêutica; 2020.

TONETTO, Isabela Fernandes de Aguiar et al. Qualidade de vida das pessoas com diabetes mellitus. Rev. esc. enferm. USP, São Paulo, v. 53, e03424, 2019. 